\title{
Study of Clinical Profile and Prognostic Factors of Acute Kidney Injury (AKI) In Tertiary Referral Centre in Marathwada
}

\author{
Dr. Sharad Garudkar ${ }^{1}$, Dr. S. G. Kulkarni ${ }^{2}$, Dr. V. S. Gulwe ${ }^{3}$ \\ ${ }^{1}$ Asst. Professor, Dept. of Medicine, P.D.V.V.P.F's Medical College, Ahmednagar. \\ ${ }^{2}$ Professor \& Head, Dept. of Nephrology, M.G.M. Medical College, Aurangabad. \\ ${ }^{3}$ Associate Professor, Dept. of Medicine, M.G.M. Medical College, Aurangabad.
}

\begin{abstract}
Mortality in acute kidney injury (AKI) remains impressively high despite technical and medical advances made following the advent of dialysis. Previous studies of prognosis in AKI have analyzed the influence of demographic factors, severity of AKI, nature of diseases causing AKI, coexisting diseases, treatment received and complications. Several other factors also affect prognosis of AKI viz. oliguria, a rise in serum creatinine greater than $3 \mathrm{mg} \%$, older debilitated patients, multiorgan failure, associated comorbid conditions, need of dialysis, suspected or proven sepsis. Hence cases of AKI were studied at tertiary health centre in Marathwada region of Maharashtra to study the clinical profile and factors affecting the prognosis of acute kidney injury.
\end{abstract}

Keywords: Acute Kidney Injury (AKI), Dialysis, Oliguria.

\section{Introduction}

The kidney is a highly vascular organ. The kidneys are important organs of our body, which deals with the excretion of the waste products of protein catabolism from the body and maintenance of water and electrolyte balance. The portion of the total cardiac output that passes through the kidneys, called as renal fraction is about $20 \%$. Kidneys are prone to develop ischemic injury whenever blood supply to them is decreased. They are also susceptible to nephrotoxic injury by virtue of their rich blood supply and the ability to concentrate toxins in the medullary interstitium and renal epithelial cells.

Acute kidney injury (AKI), previously known as acute renal failure (ARF), is a syndrome characterized by rapid decline of glomerular filtration rate (hours to weeks), retention of nitrogenous waste products and perturbation of extracellular fluid volume and electrolyte and acid base homeostasis ${ }^{1}$.

The term failure reflects only part of the spectrum of damage to the kidney that occurs clinically. In most cases of damage, the reduction in kidney function is modest. Nevertheless, this modest change has been documented to be associated with negative effects on outcome, albeit not nearly as ominous as seen with large decreases in kidney function associated with frank kidney failure that often requires acute dialysis therapies. Furthermore, the term renal is not well understood in the general population and this makes communication with patients and family more challenging; hence "kidney" has replaced "renal". Hence the name acute renal failure was changed to "acute kidney injury."

Urine output is generally reduced to $<400 \mathrm{ml} /$ day called as oliguric AKI but some patients continue to pass $>400 \mathrm{ml}$ of urine per day, called as non-oliguric AKI. In AKI nitrogenous waste products accumulate in the body and renal mechanisms responsible for maintaining water and electrolyte balance is disturbed. In addition the renal contribution to the control of acid base balance is deficient; hydrogen ions accumulate in the body producing metabolic acidosis. Loss of excretory function leads to hyperkalemia and oedema, with or without pulmonary oedema, if dietary intake of potassium and water is not restricted.

AKI is defined as any of the following ${ }^{2}$ :

1. Increase in Serum Creatinine by $0.3 \mathrm{mg} / \mathrm{dl}$ within 48 hours; or

2. Increase in Serum Creatinine to 1.5 times baseline, which is known or presumed to have occurred within the prior 7 days; or

3. Urine volume $<0.5 \mathrm{ml} / \mathrm{kg} / \mathrm{h}$ for 6 hours.

It is one of the most common clinical syndrome encountered in the clinical practice. Most AKI is reversible, the kidneys being relatively unique among major organs in its ability to recover in function.

Acute Kidney Injury is sub classified into three categories, viz prerenal, intrinsic renal and post renal failure. Prerenal failure the most common form and is characterized by renal hypoperfusion without compromising to integrity of renal parenchyma. Intrinsic renal failure is produced by disorders that directly involve renal parenchyma. These are sepsis, ischemic, various nephrotoxins and diseases of glomeruli. Post renal failure is produced by urinary tract obstruction ${ }^{1}$. 
As there are no specific therapies for treatment of ischemic and nephrotoxic AKI and mortality is so high, prevention is of paramount importance. Early identification of patients at risk with prompt elimination of potential insults is the golden rule. Aggressive restoration of intravascular volume has been shown to reduce the incidence of ARF dramatically in volume depleted states. Injudicious use of nephrotoxic drugs like aminoglycosides and NSAIDs should be avoided especially in elderly and in combination with diuretics. Dose modification should be done when renal failure has already developed. Maintenance of volume status to optimum during operative and post operative periods is very important.

Sepsis is by far the commonest cause of death in AKI, so all steps should be taken to avoid or limit it. Many a times, doctors are responsible for development of AKI in hospitals, with a little more awareness about the precipitating factors, avoidance of the injudicious use of nephrotoxic drugs, proper maintenance of intake and output records in post operative cases and a watchful readiness to act promptly if unavoidable circumstances arises, can prevent and minimize the number of AKI cases. Factors that predispose AKI are renal insult, myeloma, diabetes mellitus, proteinuria, previous cardiac or renal insufficiency, diuretic use, volume depletion, advanced age. ${ }^{3}$

Mortality rate among patients of AKI approximates 50 percent and mortality rate vary greatly, depending on the cause of $\mathrm{AKI}^{4}$.

Several factors affect prognosis of AKI viz oliguria, a rise in serum creatinine greater than $3 \mathrm{mg} \%$, older debilitated patients, multiorgan failure, associated comorbid conditions, need of dialysis, suspected or proven sepsis 5 . Hence cases of AKI were studied at Tertiary Health Centre in Marathwada region of Maharashtra MGM Medical College and Hospital, Aurangabad to study the clinical profile and factors affecting the prognosis of acute kidney injury.

\section{Observations}

Table - 1 Age And Gender Distribution

\begin{tabular}{|l|l|l|l|}
\hline Age Group & Male & Female & Total \\
\hline $0-20$ & $6(4.16)$ & $5(3.47)$ & $11(7.63)$ \\
\hline $21-40$ & $21(14.58)$ & $20(13.88)$ & $41(28.47)$ \\
\hline $41-60$ & $36(25)$ & $16(11.11)$ & $52(36.11)$ \\
\hline$\geq 61$ & $25(17.36)$ & $15(10.41)$ & $38(26.38)$ \\
\hline Total & $88(61.11)$ & $56(38.88)$ & $144(100)$ \\
\hline
\end{tabular}

Figures in ( ) indicates percentage.

Graph - 1 Age \& Gender Distribution

Male preponderance was seen in all age groups.

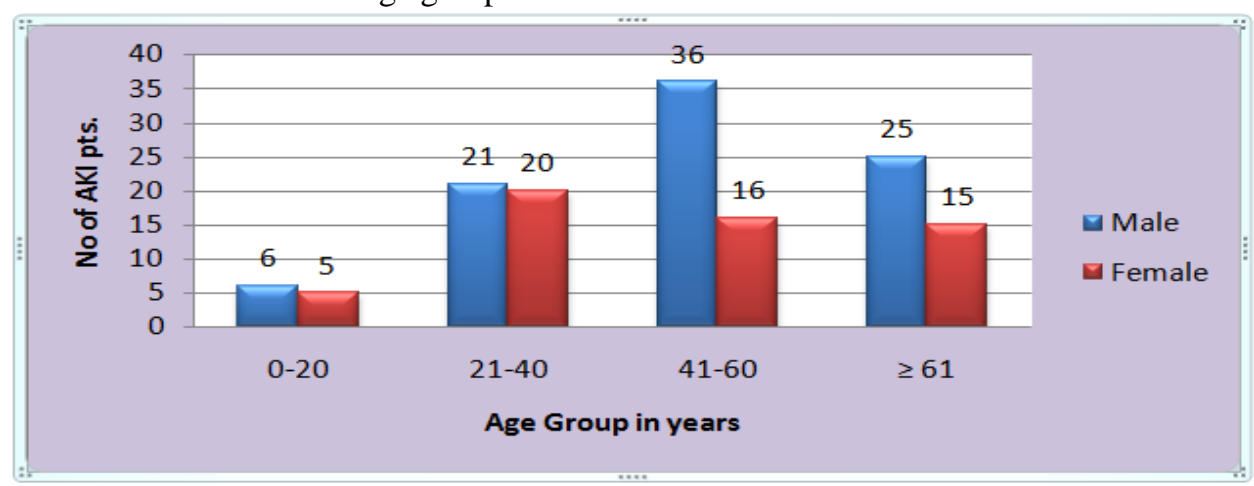

Table 2 - Etiology Of Aki

\begin{tabular}{|l|l|l|}
\hline Etiology of AKI & No. Of pts. & Percentage (\%) \\
\hline Medical AKI & 111 & 77.08 \\
\hline Obstetric AKI & 17 & 11.80 \\
\hline Surgical AKI & 16 & 11.11 \\
\hline Total & 144 & 100 \\
\hline
\end{tabular}




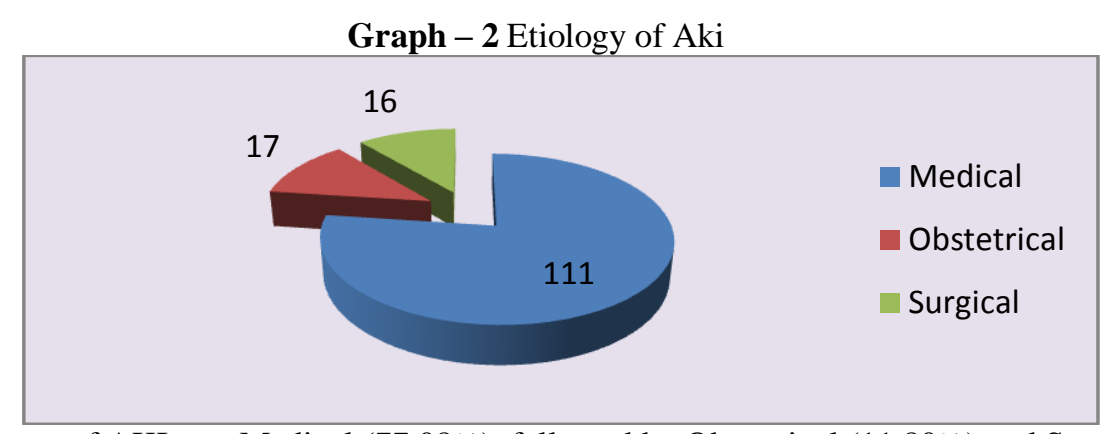

The commonest cause of AKI was Medical (77.08\%), followed by Obstetrical (11.80\%) and Surgical causes $(11.11 \%)$.

Table - 3Clinical And Laboratory Data In 144 Patients Of Aki

\begin{tabular}{|l|c|}
\hline Parameters & Mean \pm SD, Ratio \\
\hline Age (years) & $48 \pm 18.24$ \\
\hline Sex (M: F) & $88: 56(1.57: 1)$ \\
\hline Oliguria/Nonliguria & $61 / 83$ \\
\hline Hypotension & $41(28.47 \%)$ \\
\hline Bleeding tendency & $11(7.63 \%)$ \\
\hline Hyperkalemia & $40(27.77 \%)$ \\
\hline Peak Blood Urea (mg \%) & $131.50 \pm 69.94$ \\
\hline Peak serum Creatinine (mg \%) & $4.96 \pm 3.23$ \\
\hline Mortality & $29.16 \%$ \\
\hline
\end{tabular}

Out of 144 patients of AKI studied, male to female ratio was 1.57:1. Mean age was $48 \pm 18.24$. Overall mortality was $29.16 \%$.

Table - 4Causes Of Oliguric V/S Nonoliguric Aki

\begin{tabular}{|l|l|l|}
\hline Causes & $\begin{array}{l}\text { Oliguric patients } \\
(\mathrm{n}=61)\end{array}$ & $\begin{array}{l}\text { Non-oliguric } \\
\text { patients } \\
(\mathrm{n}=83)\end{array}$ \\
\hline Surgical & $11(18.03)$ & $5(6.02)$ \\
\hline Obstetrical & $5(8.19)$ & $12(14.45)$ \\
\hline Medical & $45(73.77)$ & $66(79.51)$ \\
\hline Medical causes & & \\
Sepsis & $16(35.55)$ & $20(30.30)$ \\
Acute Gastroenteritis & $6(13.33)$ & $12(18.18)$ \\
Acute Pancreatitis & $7(15.55)$ & $10(15.15)$ \\
ContrastInduced & $5(11.11)$ & $5(7.57)$ \\
Nephropathy & & \\
Hepatorenal Syndrome & $6(13.33)$ & $3(4.54)$ \\
Malaria & $2(4.44)$ & $3(4.54)$ \\
Dengue Fever & $1(2.22)$ & $4(6.06)$ \\
Snake Bite & $2(4.44)$ & $1(1.51)$ \\
Leptospirosis & $0(0)$ & $2(3.03)$ \\
HUS & $0(0)$ & $1(1.51)$ \\
& & \\
\hline
\end{tabular}

Figures in ( ) indicates percentage.

Non-oliguric AKI was more common than Oliguric AKI. Non-oliguric AKI was seen in all etiologies.

Table - 5Age And Medical Etiology Of Aki

\begin{tabular}{|l|l|l|l|l|l|l|}
\hline Age & $0-20$ & $21-40$ & $41-60$ & $\geq 61$ & Total & Percentage $(\%)$ \\
\hline Sepsis & $0(0)$ & $6(17.14)$ & $12(34.28)$ & $17(48.57)$ & $35(31.53)$ & 31.53 \\
\hline Acute Gastroenteritis & $2(11.11)$ & $3(16.66)$ & $8(44.44)$ & $5(27.77)$ & $18(16.21)$ & 16.21 \\
\hline Acute Pancreatitis & $0(0)$ & $10(62.5)$ & $6(37.5)$ & $0(0)$ & $16(14.41)$ & 14.41 \\
\hline $\begin{array}{l}\text { Contrast Induced } \\
\text { Nephropathy }\end{array}$ & $0(0)$ & $1(10)$ & $4(40)$ & $5(50)$ & $10(9)$ & 9 \\
\hline $\begin{array}{l}\text { Hepatorenal } \\
\text { Syndrome }\end{array}$ & $1(11.11)$ & $0(0)$ & $4(44.44)$ & $4(44.44)$ & $9(8.10)$ & 8.10 \\
\hline Drug Induced & $1(14.28)$ & $0(0)$ & $5(71.42)$ & $1(14.28)$ & $7(6.30)$ & 6.30 \\
\hline Malaria & $1(20)$ & $3(60)$ & $1(20)$ & $0(0)$ & $5(4.50)$ & 4.50 \\
\hline Dengue Fever & $0(0)$ & $2(40)$ & $1(20)$ & $2(40)$ & $5(4.50)$ & 4.50 \\
\hline Snake Bite & $1(33.33)$ & $1(33.33)$ & $1(33.33)$ & $0(0)$ & $3(2.70)$ & 2.70 \\
\hline
\end{tabular}


Study of clinical profile and prognostic factors of Acute Kidney Injury (AKI) in Tertiary Referral...

\begin{tabular}{|l|l|l|l|l|l|l|}
\hline Leptospirosis & $0(0)$ & $0(0)$ & $2(100)$ & $0(0)$ & $2(1.80)$ & 1.80 \\
\hline HUS & $1(100)$ & $0(0)$ & $0(0)$ & $0(0)$ & $1(0.69)$ & 0.69 \\
\hline Total & $7(6.30)$ & $26(23.42)$ & $44(39.63)$ & $34(30.63)$ & $111(100)$ & 100 \\
\hline
\end{tabular}

Figures in ( ) indicates percentage.

AKI secondary to sepsis and contrast induced nephropathy were common in age group more than 61 years. AKI secondary to acute pancreatitis was more common in age group 21-40.

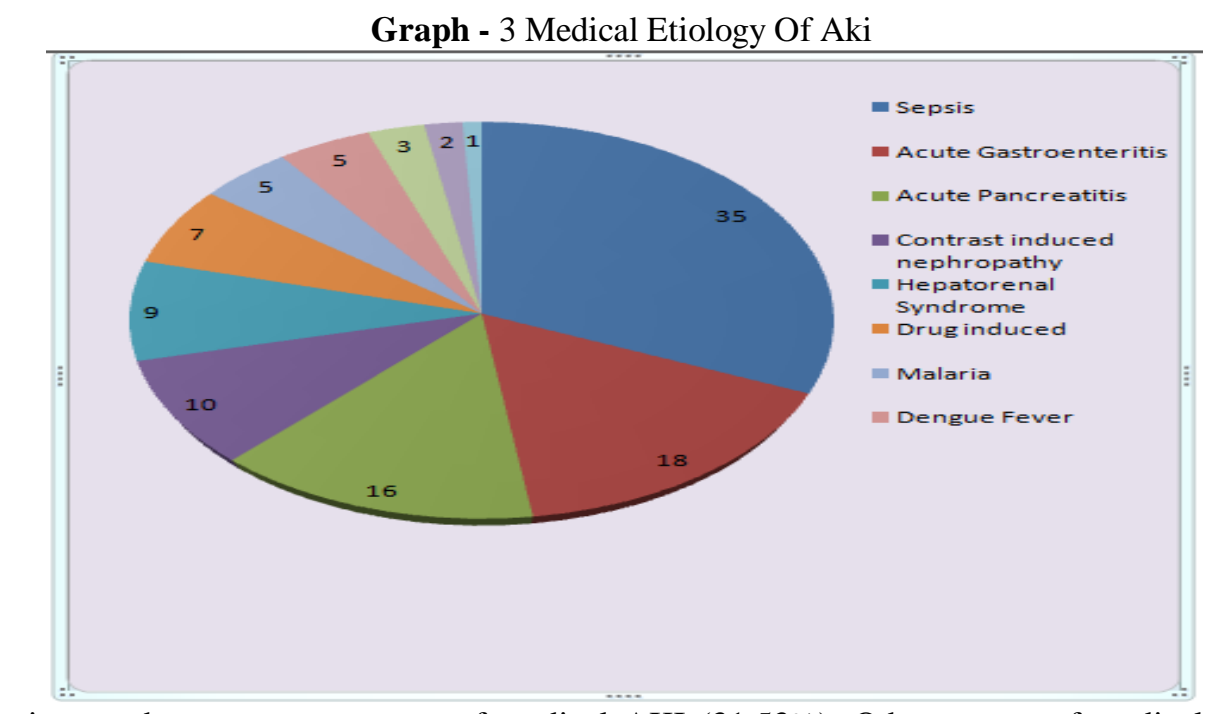

Sepsis was the commonest cause of medical AKI (31.53\%). Other causes of medical AKI were as follows: Acute Gastroenteritis (16.21\%), Acute pancreatitis (14.41\%), Contrast induced nephropathy (9\%), Hepatorenal syndrome (8.10\%), Drug induced (6.30\%), Malaria (4.50\%), Dengue fever (4.50\%), Snake bite (2.70\%), Leptospirosis (1.80\%), HUS (0.69\%).

Table-6Age And Etiology Of Aki

\begin{tabular}{|l|l|l|l|l|}
\hline Age & Medical & Obstetric & Surgical & Total \\
\hline $0-20$ & $7(4.86)$ & $4(2.77)$ & $0(0)$ & $11(7.63)$ \\
\hline $21-40$ & $26(18.05)$ & $13(9.02)$ & $2(1.38)$ & $41(28.47)$ \\
\hline $41-60$ & $44(30.55)$ & $0(0)$ & $8(5.55)$ & $52(36.11)$ \\
\hline$\geq 61$ & $34(23.61)$ & $0(0)$ & $6(4.16)$ & $40(27.77)$ \\
\hline Total & $111(77.08)$ & $17(11.80)$ & $16(11.11)$ & $144(100)$ \\
\hline
\end{tabular}

Figures in ( ) indicates percentage.

AKI due to medical and surgical causes were more common in patients with age more than 41 years. AKI due to obstetric causes was more common in age group 21-40 years.

Table - 7 Relation Of Age Distribution To Mortality

\begin{tabular}{|l|l|l|l|l|}
\hline \multicolumn{1}{|c|}{ Age (yrs) } & Survival & Mortality & Total patients & Percentage Mortality \\
\hline $0-20$ & $9(90)$ & $1(10)$ & $10(6.94)$ & 10 \\
\hline $21-40$ & $28(68.29)$ & $13(31.70)$ & $41(28.47)$ & 31.70 \\
\hline $41-60$ & $40(75.47)$ & $13(24.52)$ & $53(36.80)$ & 24.52 \\
\hline$\geq 61$ & $25(62.5)$ & $15(37.5)$ & $40(27.77)$ & 37.5 \\
\hline Total & $102(70.83)$ & $42(29.16)$ & $144(100)$ & 29.16 \\
\hline
\end{tabular}

$($ Chi square $=2.79) \quad \mathrm{p}>0.05$ (non-significant) 
GRAPH 4 - Mortality According To Age Group

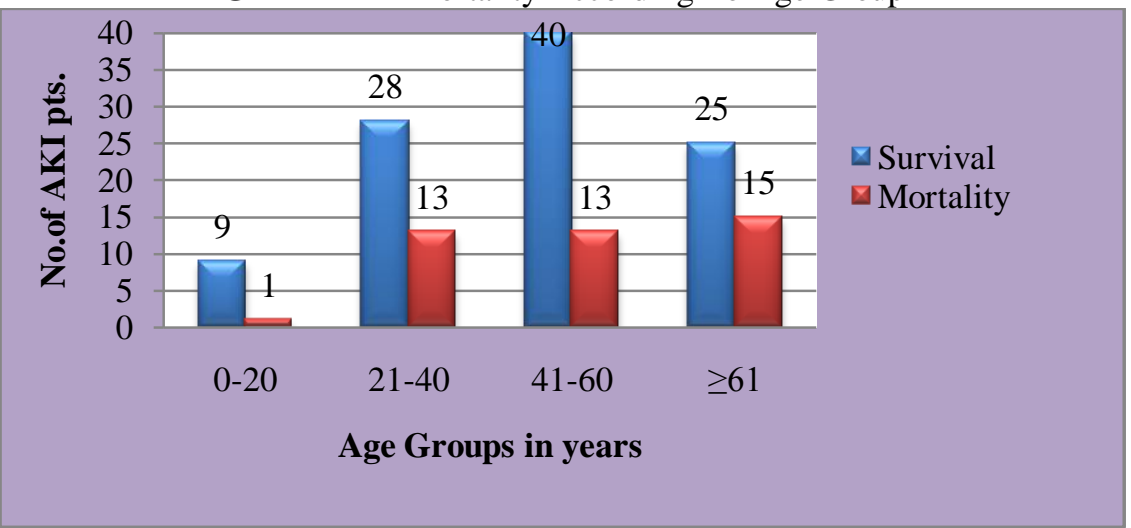

Although the mortality was maximum (37.5\%) in age group more than 61years as compared to other age groups, chi square test showed no difference in mortality among various age groups. Overall mortality was $29.16 \%$.

TABLE - 8: Relation Of Gender Distribution To Mortality

\begin{tabular}{|l|l|l|l|l|}
\hline Gender & Survival & Mortality & Total patient & Percentage Mortality \\
\hline Male & $61(69.31)$ & $27(30.68)$ & $88(61.11)$ & 30.68 \\
\hline Female & $41(73.21)$ & $15(26.78)$ & $56(38.88)$ & 26.78 \\
\hline Total & $102(70.83)$ & $42(29.16)$ & $144(100)$ & 29.16 \\
\hline
\end{tabular}

Figures in () indicates percentage. $($ Chi square $=0.2517) \quad \mathrm{p}>0.05$ (non-significant)

GRAPH - 5 Gender Distribution To Mortality

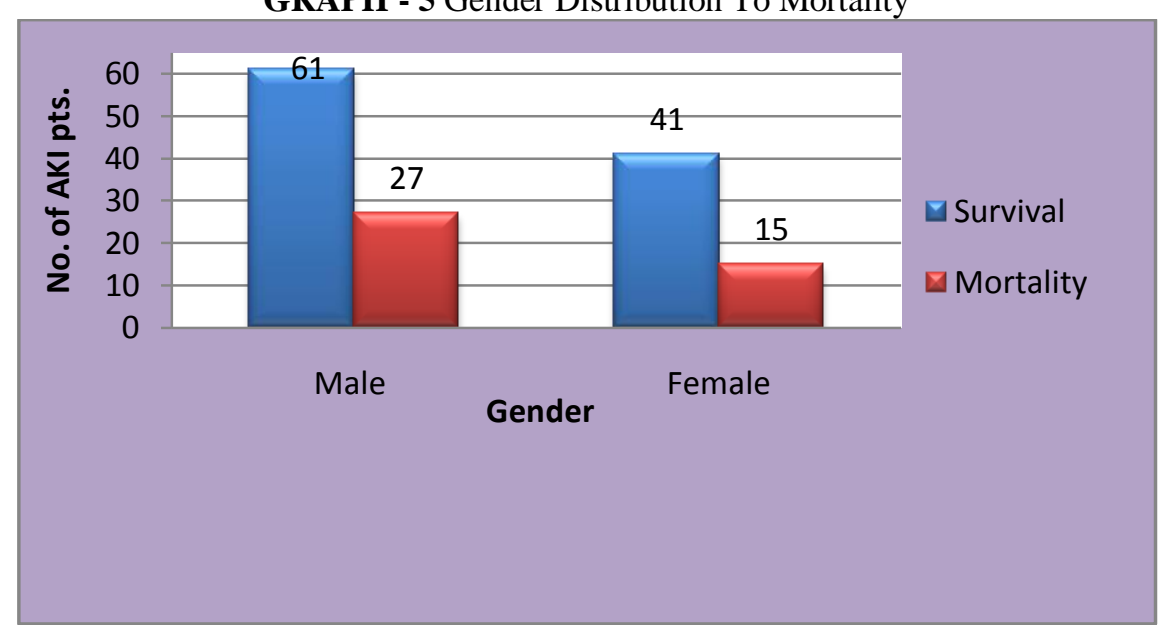

Although mortality was more in males (30.68\%) than females (26.78\%) but statistically there was no significant difference in mortality.

TABLE - 9: Relation Of Etiology Of Aki To Mortality

\begin{tabular}{|l|l|l|l|l|}
\hline Etiology & Survival & Mortality & Total Patients & Percentage Mortality \\
\hline Medical & $80(72.07)$ & $31(27.92)$ & $111(77.08)$ & 27.92 \\
\hline Obstetric & $13(76.47)$ & $4(23.52)$ & $17(11.80)$ & 23.52 \\
\hline Surgical & $9(56.25)$ & $7(43.75)$ & $16(11.11)$ & 43.75 \\
\hline Total & $102(70.83)$ & $42(29.16)$ & $144(100)$ & 29.16 \\
\hline
\end{tabular}

Figure in () indicates percentage $\quad($ Chi square $=1.995) \quad p>0.05$ (Non Significant) 


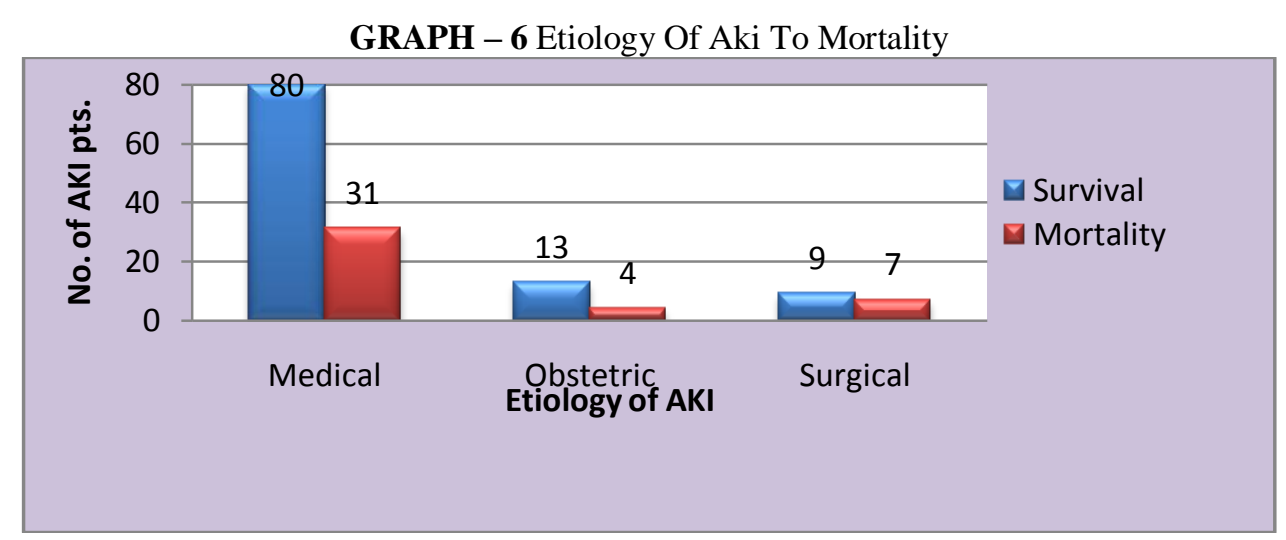

The mortality was more in surgical causes of AKI $(43.75 \%)$ than medical $(27.92 \%)$ or obstetrical causes (23.52\%) of AKI, but the difference in mortality among these three groups was statistically not significant.

TABLE - 10: Relation Of Medical Causes Of Aki To Mortality

\begin{tabular}{|l|l|l|l|l|}
\hline Etiology & Survival & Mortality & Total & Percentage Mortality \\
\hline Sepsis & $25(71.42)$ & $10(28.57)$ & $35(31.53)$ & 28.57 \\
\hline Acute Gastroenteritis & $17(94.44)$ & $1(5.55)$ & $18(16.21)$ & 5.55 \\
\hline Acute Pancreatitis & $8(50)$ & $8(50)$ & $16(14.41)$ & 50 \\
\hline Contrast Induced Nephropathy & $5(50)$ & $5(50)$ & $10(9)$ & 50 \\
\hline Hepatorenal Syndrome & $6(66.66)$ & $3(33.33)$ & $9(8.10)$ & 33.33 \\
\hline Drug Induced AKI & $6(85.71)$ & $1(14.28)$ & $7(6.30)$ & 14.28 \\
\hline Malaria & $4(80)$ & $1(20)$ & $5(4.50)$ & 20 \\
\hline Dengue fever & $3(60)$ & $2(40)$ & $5(4.50)$ & 40 \\
\hline Snakebite & $3(100)$ & $0(0)$ & $3(2.70)$ & 0 \\
\hline Leptospirosis & $2(100)$ & $0(0)$ & $2(1.80)$ & 0 \\
\hline HUS & $1(100)$ & $0(0)$ & $1(0.90)$ & 0 \\
\hline Total & $80(72.07)$ & $31(27.92)$ & $111(100)$ & 27.92 \\
\hline
\end{tabular}

Among medical causes of AKI highest mortality was seen in AKI secondary to Acute Pancreatitis $(50 \%)$ and Contrast induced nephropathy (50\%) followed by Hepatorenal syndrome (33.33\%). Mortality in sepsis induced AKI was $29.57 \%$. Overall mortality in medical causes of AKI was $27.92 \%$. High mortality in contrast induced nephropathy was due to associated multiple comorbid conditions were present in $70 \%$ of contrast induced nephropathy cases.

TABLE - 11: Relation Of Obstetric Causes Of Aki To Mortality

\begin{tabular}{|l|l|l|l|l|}
\hline Etiology & Survival & Mortality & Total Patients & Percentage Mortality \\
\hline Puerperal sepsis & $6(66.66)$ & $3(33.33)$ & $9(52.94)$ & 33.33 \\
\hline Eclampsia & $4(100)$ & $0(0)$ & $4(23.52)$ & 0 \\
\hline PPH & $1(50)$ & $1(50)$ & $2(11.76)$ & 50 \\
\hline Abortion & $2(100)$ & $0(0)$ & $2(11.76)$ & 0 \\
\hline Total & $13(76.47)$ & $4(23.52)$ & $17(100)$ & 23.52 \\
\hline
\end{tabular}

Figures in $($ ) indicates percentage. (Chi square $=3.1207) \quad \mathrm{p}>0.05$ (non-significant)

Puerperal sepsis was the major cause of Obstetric AKI (52.94\%). Among obstetric causes maximum mortality was seen in PPH (50\%). But the difference in mortality among various causes of obstetric AKI was statistically non-significant. Overall mortality in obstetric AKI was $23.52 \%$.

TABLE - 12: Relation Of Surgical Causes Of Aki To Mortality

\begin{tabular}{|l|l|l|l|l|}
\hline Etiology & Survival & Mortality & Total & $\begin{array}{l}\text { Percentage } \\
\text { Mortality }\end{array}$ \\
\hline Postoperative & $3(42.85)$ & $4(57.14)$ & $7(43.75)$ & 57.14 \\
\hline $\begin{array}{l}\text { Obstructive } \\
\text { Uropathy }\end{array}$ & $5(71.42)$ & $2(28.57)$ & $7(43.75)$ & 28.57 \\
\hline Post-traumatic & $1(50)$ & $1(50)$ & $2(12.5)$ & 50 \\
\hline Total & $9(56.25)$ & $7(43.75)$ & $16(100)$ & 43.75 \\
\hline
\end{tabular}

Figures in () indicates percentage $($ Chi square $=2.47) \quad \mathrm{p}>0.05$ (non-significant) 
Study of clinical profile and prognostic factors of Acute Kidney Injury (AKI) in Tertiary Referral...

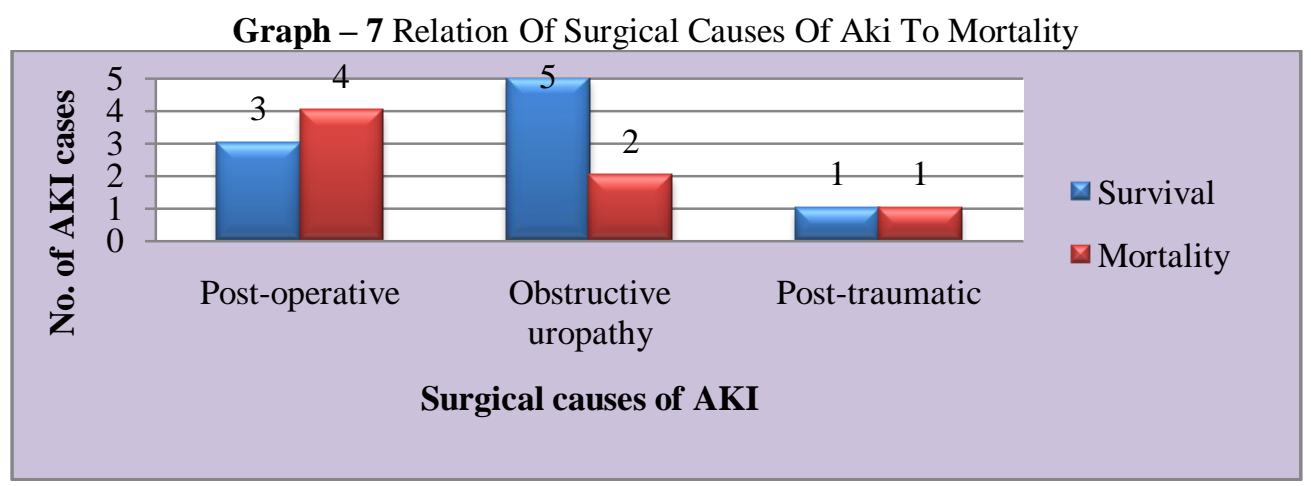

Mortality was more in post-operative cause of surgical AKI (57.14\%) than other causes of surgical AKI, but it was statistically non-significant. The overall mortality in surgical group of AKI was $43.75 \%$.

TABLE - 13: Relation Of Type Of Aki To Mortality

\begin{tabular}{|l|l|l|l|l|}
\hline Type of AKI & Survival & Mortality & Total Patients & $\begin{array}{l}\text { Percentage } \\
\text { Mortality }\end{array}$ \\
\hline Oliguric & $32(52.45)$ & $29(47.54)$ & $61(42.36)$ & 47.54 \\
\hline Non-oliguric & $70(84.33)$ & $13(15.66)$ & $83(57.63)$ & 15.66 \\
\hline Total & $102(70.83)$ & $42(29.16)$ & $144(100)$ & 29.16 \\
\hline
\end{tabular}

Figures in $($ ) indicates percentage. $\quad($ Chi square $=17.28) \quad \mathrm{p}<0.001$ (Significant)

GRAPH 8 - Relation Of Type Of Aki To Mortality

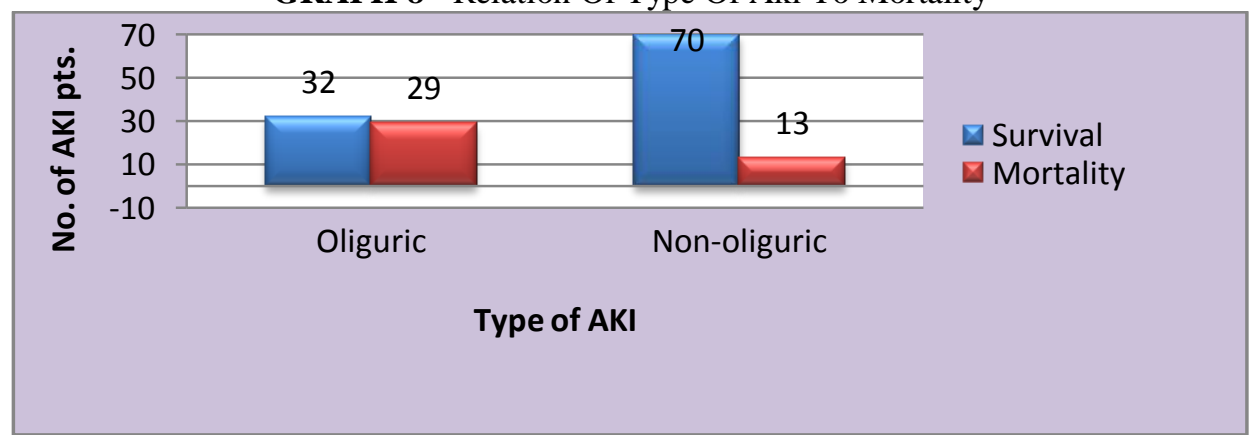

Non-oliguric AKI (57.63\%) was more common than oliguric AKI (42.36\%). Mortality was more in oliguric AKI (47.54\%) than in non-oliguric AKI (15.66\%). This difference in mortality among oliguric and nonoliguric AKI was statistically significant.

TABLE - 14 Relation Of Mode Of Treatment To Mortality

\begin{tabular}{|l|l|l|l|l|}
\hline Mode of treatment & Survival & Mortality & Total & Percentage Mortality \\
\hline Conservative & $74(75.51)$ & $24(24.49)$ & $98(68.05)$ & 24.49 \\
\hline Dialysis & $28(60.87)$ & $18(39.13)$ & $46(31.94)$ & 39.13 \\
\hline Total & $102(70.83)$ & $42(29.16)$ & $144(100)$ & 29.16 \\
\hline
\end{tabular}

Figures in ( ) indicates percentage. $\quad$ (Chi square $=3.24) \quad \mathrm{p}<0.05$ (Significant)

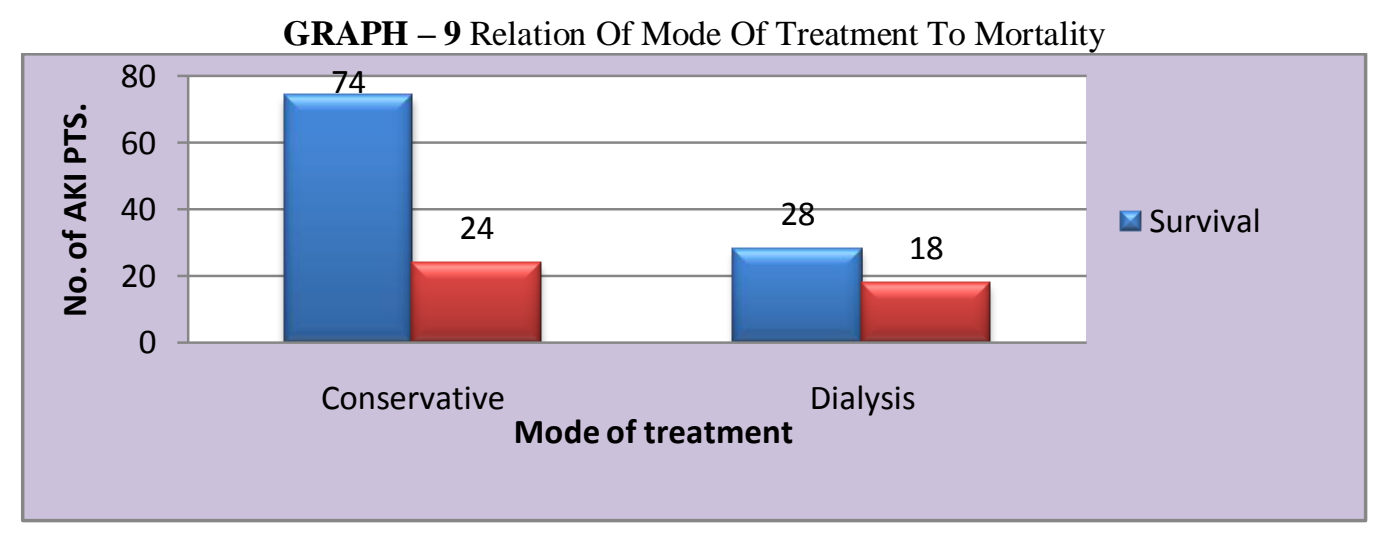


Study of clinical profile and prognostic factors of Acute Kidney Injury (AKI) in Tertiary Referral...

Mortality in patients who needed dialysis was $39.13 \%$ while mortality in patients who were treated conservatively was $24.49 \%$. This difference in mortality was statistically significant. Thus mortality was more in patients who needed dialysis.

TABLE - 15: Relation Of Mode Of Treatment To Mortality

\begin{tabular}{|l|l|l|l|l|}
\hline Mode of treatment & Survival & Mortality & Total Patients & $\begin{array}{l}\text { Percentage } \\
\text { Mortality }\end{array}$ \\
\hline Conservative & $74(75.51)$ & $24(24.49)$ & $98(68.05)$ & 24.49 \\
\hline Hemodialysis & $27(72.97)$ & $10(27.02)$ & $37(25.69)$ & 27.02 \\
\hline Peritoneal Dialysis & $2(20)$ & $8(80)$ & $10(6.94)$ & 80 \\
\hline Total & $102(70.83)$ & $42(29.16)$ & $144(100)$ & 29.16 \\
\hline
\end{tabular}

Figures in () indicates percentage. $\quad$ (Chi square $=13.66)$ $\mathrm{p}<0.001$ (Significant)

GRAPH 10 - Relation Of Mode Of Treatment To Mortality

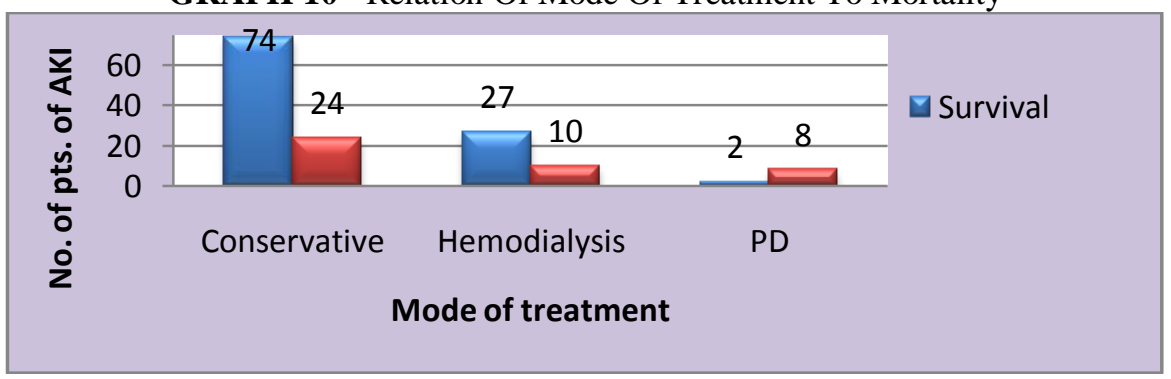

Majority of patients were treated conservatively $(68.05 \%)$. Mortality was more in patients treated with peritoneal dialysis $(80 \%)$ than in patients treated conservatively $(24.49 \%)$ and with hemodialysis $(27.02 \%)$. This different in mortality with different modes of treatment was statistically significant. This was because in our institute peritoneal dialysis was considered in patients who were hemodynamically not suitable for hemodialysis.

TABLE - 16 Relation Of Time Of Intervention To Mortality

\begin{tabular}{|l|l|l|l|l|}
\hline \multicolumn{1}{|c|}{ Time } & Survival & Mortality & Total Patients & Percentage Mortality \\
\hline Within 48 hrs & $36(83.72)$ & $7(16.27)$ & $43(29.86)$ & 16.27 \\
\hline After 48 hrs & $66(65.34)$ & $35(34.65)$ & $101(70.13)$ & 34.65 \\
\hline Total & $102(70.83)$ & $42(29.16)$ & $144(100)$ & 29.16 \\
\hline
\end{tabular}

Figures in ( ) indicates percentage. (Chi square $=4.94) \quad \mathrm{p}<0.05$ (Significant)

GRAPH 11 - Relation Of Time Of Intervention To Mortality

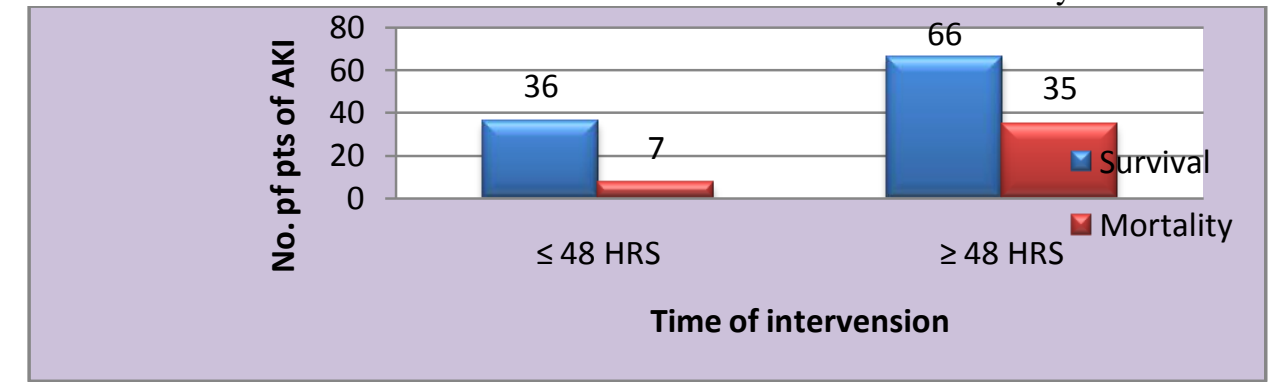

Majority of patients were treated after $48 \mathrm{hrs}$ of diagnosis of AKI. Mortality was more in patients treated after 48 hours of diagnosis of AKI $(34.65 \%)$ as compared with patients treated within 48 hours of diagnosis of AKI (16.27\%). This difference in mortality due to time of intervention was statistically significant.

TABLE - 17: Relation Of Peak Serum Creatinine Level To Mortality

\begin{tabular}{|l|l|l|l|l|}
\hline $\begin{array}{l}\text { Peak Serum } \\
\text { Creatinine (mg \%) }\end{array}$ & Survival & Mortality & Total Patients & Percentage Mortality \\
\hline$<3$ & $36(87.80)$ & $5(12.19)$ & $41(28.47)$ & 12.19 \\
\hline$\geq 3$ & $66(64.07)$ & $37(35.92)$ & $103(71.52)$ & 35.92 \\
\hline Total & $102(70.83)$ & $42(29.16)$ & $144(100)$ & 29.16 \\
\hline
\end{tabular}

Figures in ( ) indicates percentage. $\quad($ Chi square $=7.98) \quad \mathrm{p}<0.05$ (Significant) 
GRAPH 12 - Relation Of Peak Serum Creatinine Level To Mortality

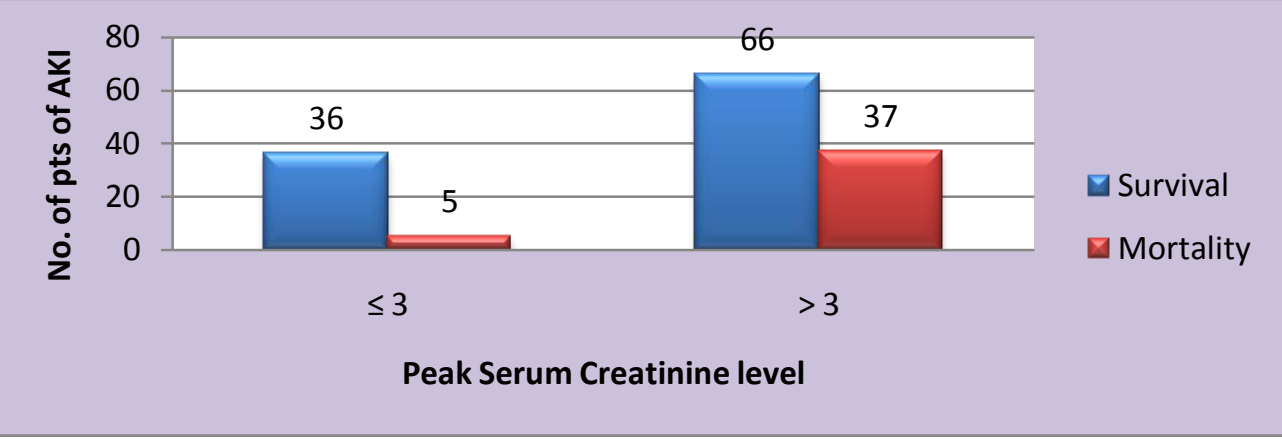

Majority of patients had peak serum creatinine more than $3 \mathrm{mg} \%(71.52 \%)$. Mortality was more in patients who had peak serum creatinine more than $3 \mathrm{mg} \%(35.92 \%)$ than in patients who had peak serum creatinine less than $3 \mathrm{mg} \%$ (12.19\%). This difference in mortality was statistically significant.

TABLE - 18: Relation Of Peak Blood Urea Level To Mortality

\begin{tabular}{|l|l|l|l|l|}
\hline $\begin{array}{l}\text { Peak Blood Urea } \\
\text { level }(\mathrm{mg} \%)\end{array}$ & Survival & Mortality & Total Patients & $\begin{array}{l}\text { Percentage } \\
\text { Mortality }\end{array}$ \\
\hline$<100$ & $44(81.48)$ & $10(18.51)$ & $54(37.5)$ & 18.51 \\
\hline$\geq 100$ & $58(64.44)$ & $32(35.55)$ & $90(62.5)$ & 35.55 \\
\hline Total & $102(70.83)$ & $42(29.16)$ & $144(100)$ & 29.16 \\
\hline
\end{tabular}

Figures in ( ) indicates percentage. (Chi square $=4.74) \quad \mathrm{p}<0.05$ (Significant)

GRAPH 13 - Relation Of Peak Blood Urea Level To Mortality

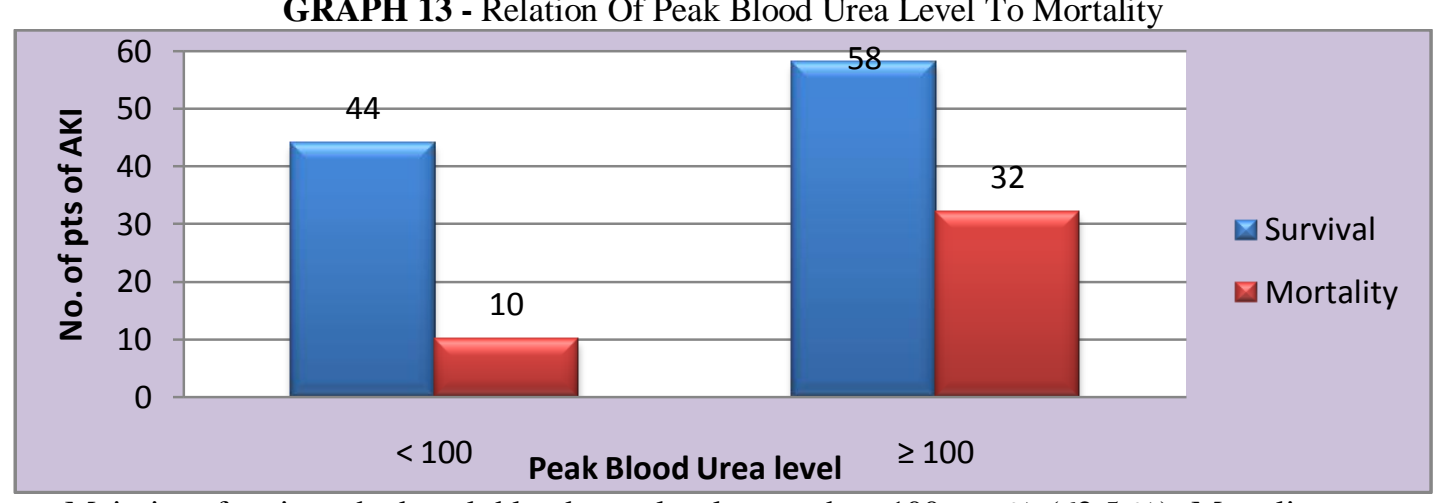

Majority of patients had peak blood urea level more than $100 \mathrm{mg} \%$ (62.5\%). Mortality was more in patients who had peak blood urea level more than $100 \mathrm{mg} \%(35.55 \%)$ as compared to patients who had peak blood urea level less than $100 \mathrm{mg} \%(18.51 \%)$. This difference in mortality was statistically significant.

TABLE - 19: Relation Of Serum Sodium Level To Mortality

\begin{tabular}{|l|l|l|l|l|}
\hline $\begin{array}{l}\text { Serum } \\
(\mathrm{meq} / \mathrm{L})\end{array}$ & Sodium & Mortality & Total Patients & Percentage Mortality \\
\hline$<136$ & $57(75)$ & $19(25)$ & $76(52.77)$ & 25 \\
\hline$\geq 136$ & $45(66.17)$ & $23(33.82)$ & $68(47.22)$ & 33.82 \\
\hline Total & $102(70.83)$ & $42(29.16)$ & $144(100)$ & 29.16 \\
\hline
\end{tabular}

Figures in ( ) indicates percentage. (Chi square $=1.35) \quad \mathrm{p}>0.05$ (Non Significant)

Hyponatremia was seen in 76 patients $(52.77 \%)$. There was no difference in AKI mortality among patients with or without hyponatremia.

TABLE - 20: Relation Of Serum Potassium Level To Mortality

\begin{tabular}{|l|l|l|l|l|}
\hline $\begin{array}{l}\text { Serum Potassium } \\
\text { level (meq/L) }\end{array}$ & Survival & Mortality & Total Patients & Percentage Mortality \\
\hline$\leq 5.0$ & $75(72.81)$ & $29(28.15)$ & $103(71.52)$ & 28.15 \\
\hline$>5.0$ & $27(67.5)$ & $13(31.70)$ & $40(27.77)$ & 31.70 \\
\hline Total & $102(70.83)$ & $42(29.16)$ & $144(100)$ & 29.16 \\
\hline
\end{tabular}

Figures in ( ) indicates percentage (Chi square $=0.4924) \quad p>0.05$ (Non Significant)

Hyperkalemia was seen in 40 patients $(27.77 \%)$. There was no difference in mortality in patients with or without hyperkalemia. 
Study of clinical profile and prognostic factors of Acute Kidney Injury (AKI) in Tertiary Referral...

TABLE - 21: Relation Of Serum Bilirubin Level To Mortality

\begin{tabular}{|l|l|l|l|l|}
\hline $\begin{array}{l}\text { Serum Bilirubin } \\
\text { level (meq/L) }\end{array}$ & Survival & Mortality & Total Patients & Percentage Mortality \\
\hline$<2$ & $73(73)$ & $27(27)$ & $100(69.44)$ & 27 \\
\hline$\geq 2$ & $29(65.90)$ & $15(34.09)$ & $44(30.55)$ & 34.09 \\
\hline Total & $102(70.83)$ & $42(29.16)$ & $144(100)$ & 29.16 \\
\hline
\end{tabular}

Figures in ( ) indicates percentage. (Chi square $=0.7431) \quad \mathrm{p}>0.05$ (Non Significant)

Serum bilirubin more than $2 \mathrm{mg} \%$ was seen in 44 patients $(30.55 \%)$. Hyperbilirubinemia had statistically no significant effect on mortality.

TABLE - 22 : Relation Of Platelet Level To Mortality

\begin{tabular}{|l|l|l|l|l|}
\hline $\begin{array}{l}\text { Serum Platelet level } \\
(/ \mathrm{mm} 3)\end{array}$ & Survival & Mortality & Total Patients & Percentage Mortality \\
\hline$<50,000$ & $14(60.87)$ & $9(39.13)$ & $23(15.97)$ & 39.13 \\
\hline$\geq 50,000$ & $88(72.72)$ & $33(27.27)$ & $121(84.02)$ & 27.27 \\
\hline Total & $102(70.83)$ & $42(29.16)$ & $144(100)$ & 29.16 \\
\hline
\end{tabular}

Figures in ( ) indicates percentage. (Chi Square $=0.7844) \mathrm{p}>0.05$ (Non Significant)

Platelet count less than 50,000 was seen in 23 patients $(15.97 \%)$. There was no statistically significant difference in mortality in AKI patients with or without thrombocytopenia.

TABLE - 23: Relation Of Total Leucocyte Count To Mortality

\begin{tabular}{|l|l|l|l|l|}
\hline $\begin{array}{l}\text { Total Leucocyte } \\
\text { Count }(/ \mathrm{mm} 3)\end{array}$ & Survival & Mortality & Total Patients & Percentage Mortality \\
\hline$<11000$ & $30(73.17)$ & $11(26.82)$ & $41(28.47)$ & 26.82 \\
\hline$\geq 11000$ & $72(69.90)$ & $31(30.09)$ & $103(71.52)$ & 30.09 \\
\hline Total & $102(70.83)$ & $42(29.16)$ & $144(100)$ & 29.16 \\
\hline
\end{tabular}

Figures in ( ) indicates percentage. (Chi square $=0.1501) \quad \mathrm{p}>0.05$ (Non Significant)

Leucocytosis (TLC $\geq 11,000 / \mathrm{cmm})$ was seen in 103 patients $(71.52 \%)$. Mortality was more in patients with leucocytosis $(30.09 \%)$ as compared with patients without leucocytosis $(26.82 \%)$, but the difference in mortality was statistically insignificant.

TABLE - 24: Relation Of Bleeding Tendency To Mortality

\begin{tabular}{|l|l|l|l|l|}
\hline Bleeding Tendency & Survival & Mortality & Total Patients & Percentage Mortality \\
\hline Present & $5(45.45)$ & $6(54.54)$ & $11(7.63)$ & 54.54 \\
\hline Absent & $97(72.93)$ & $36(27.06)$ & 133() & 27.06 \\
\hline Total & $102(70,83)$ & $42(29.16)$ & $144(100)$ & 29.16 \\
\hline
\end{tabular}

Figures in ( ) indicates percentage. $\quad($ Chi square $=3.73) \quad \mathrm{p}<0.05$ (Significant)

Bleeding tendency was seen in 11 patients $(7.63 \%)$. Mortality was more in patients who had bleeding tendency $(54.54 \%)$ as compared with patients without bleeding tendency $(27.06 \%)$. This difference in mortality was statistically significant.

TABLE - 25: Relation Of Hypotension To Aki Mortality

\begin{tabular}{|l|l|l|l|l|}
\hline Hypotension & Survival & Mortality & Total Patients & Percentage Mortality \\
\hline Present & $13(31.70)$ & $28(68.29)$ & $41(28.47)$ & 68.29 \\
\hline Absent & $89(86.40)$ & $14(13.59)$ & $103(71.52)$ & 13.59 \\
\hline Total & $102(70.83)$ & $42(29.16)$ & $144(100)$ & 29.16 \\
\hline
\end{tabular}

Figures in ( ) indicates percentage. $\quad($ Chi square $=42.49) \quad \mathrm{p}<0.001$ (Significant)

Hypotension was seen in 41 patients $(28.47 \%)$. Mortality was more in patients who had hypotension $(68.29 \%)$ than in patients without hypotension $(13.59 \%)$. This difference in the mortality was statistically significant.

TABLE - 26: Relation Of Other Organ Involvement (Associated Comorbid Condition To Aki Mortality)

\begin{tabular}{|l|l|l|l|l|}
\hline Other organ involvement & Survival & Mortality & Total Patients & $\begin{array}{l}\text { Percentage } \\
\text { Mortality }\end{array}$ \\
\hline Present & $36(53.73)$ & $31(46.26)$ & $67(46.52)$ & 46.26 \\
\multicolumn{1}{|c|}{ Respiratory } & $10(38.46)$ & $16(61.53)$ & $26(38.80)$ & 61.53 \\
Hepatic & $22(62.85)$ & $13(37.14)$ & $35(52.23)$ & 37.14 \\
CNS & $4(66.66)$ & $2(33.33)$ & $6(8.95)$ & 33.33 \\
\hline Absent & $66(85.71)$ & $11(14.28)$ & $77(53.47)$ & 14.28 \\
\hline Total & $102(70.83)$ & $42(29.16)$ & $144(100)$ & 29.16 \\
\hline
\end{tabular}


Figures in ( ) indicates percentage. (Chi square $=17.73$ )

$\mathrm{p}<0.001$ (Significant)

Associated comorbid conditions were seen in 67 patients (46.52\%). Associated comorbid conditions like hepatic, respiratory failure or CNS involvement increased the mortality in AKI. This difference in mortality was statistically significant.

TABLE -27complications Related To Etiology Of Aki

\begin{tabular}{|l|l|l|l|l|l|}
\hline Etiology & Hypotension & $\begin{array}{l}\text { Bleeding } \\
\text { Tendency }\end{array}$ & Hyperkalemia & $\begin{array}{l}\text { Respiratory } \\
\text { failure }\end{array}$ & CNS (Encephalopathy) \\
\hline Medical & & & & & \\
\hline Sepsis & 13 & 2 & 9 & 9 & 2 \\
\hline $\begin{array}{l}\text { Acute } \\
\text { Gastroenteritis }\end{array}$ & 0 & 0 & 6 & 0 & 3 \\
\hline Acute Pancreatitis & 6 & 1 & 7 & 2 & 0 \\
\hline $\begin{array}{l}\text { Contrast Induced } \\
\text { Nephropathy }\end{array}$ & 3 & 1 & 3 & 5 & 1 \\
\hline $\begin{array}{l}\text { Hepatorenal } \\
\text { Syndrome }\end{array}$ & 4 & 2 & 1 & 0 & 0 \\
\hline Drug Induced & 1 & 0 & 4 & 1 & 0 \\
\hline Malaria & 1 & 0 & 1 & 0 \\
\hline Dengue Fever & 1 & 0 & 1 & 1 & 0 \\
\hline Snake Bite & 1 & 1 & 0 & 0 & 0 \\
\hline Leptospirosis & 0 & 2 & 0 & 0 & 0 \\
\hline HUS & 0 & 0 & 0 & 0 & 0 \\
\hline Obstetrical & 5 & 0 & 6 & 2 & 0 \\
\hline Surgical & 6 & 2 & 3 & 5 & 0 \\
\hline Total(144) & $41(28.47 \%)$ & $11(7.63 \%)$ & $40(27.77 \%)$ & $26(18.05 \%)$ & $6(4.16 \%)$ \\
\hline
\end{tabular}

Hypotension (28.47\%) was the commonest complication of AKI followed by hyperkalemia (27.77\%).

TABLE - 28recovery Of Renal Function

\begin{tabular}{|l|l|l|}
\hline Recovery & Total Patients & Percentage \\
\hline Mortality & 42 & 29.16 \\
\hline Incomplete recovery & 6 & 4.16 \\
\hline Complete recovery & 96 & 66.66 \\
\hline
\end{tabular}

Most of the patients had complete recovery from AKI (66.66\%). Incomplete recovery was observed in (6/144) $4.16 \%$ of patients.

\section{Conclusion}

Total 144 cases of AKI were studied at Tertiary Health Centre (MGM Medical College and Hospital, Aurangabad) of Marathwada region of Maharashtra, during the period from June 2011 to Nov 2013 to study clinical profile, etiology and prognostic factors of AKI.

Majority of cases were in the age group 41-60 years and male to female ratio was 1.57:1. Medical causes of AKI were more common than obstetrical and surgical. In medical causes sepsis was the most common cause. In medical causes, Acute Pancreatitis and Contrast induced nephropathy had the highest mortality (50\%). High mortality in contrast induced nephropathy in present study was due to $70 \%$ patients of CI-AKI had associated multiple comorbid conditions, $80 \%$ patients of CI-AKI had baseline serum creatinine more than 3 $\mathrm{mg} \%$ and $40 \%$ patients needed dialysis. The mortality was more in surgical AKI than medical and obstetric AKI, but the difference in mortality among surgical, medical and obstetrical AKI was statistically not significant. Sepsis and contrast induced nephropathy were more common in age more than 61 years. Hypotension, hyperkalemia, bleeding tendency and respiratory failure were the common complications observed. Non-oliguric AKI was more common than oliguric AKI. Non-oliguric AKI was seen in all etiologies. Mortality in oliguric AKI was more than nonoliguric AKI. Mean peak blood urea and Sr. creatinine level were $131.50 \pm 69.94 \mathrm{mg} \%$ and $4.96 \pm 3.23 \mathrm{mg} \%$ respectively. Overall mortality was $29.16 \%$. Maximum mortality was seen in patients with age more than 61 years but there was no statistically significant difference in mortality among various age groups. Thus age was a weak determinant of mortality in AKI. Mortality was more in males than females however, this was not statistically significant. Thus gender was a weak determinant of mortality in AKI. In obstetrical AKI, Puerperal Sepsis was the commonest cause of AKI (52.94\%). Overall mortality in obstetric cases was $17.64 \%$. There was no significant statistical difference in mortality among obstetric causes of AKI. In surgical AKI, post-operative AKI and obstructive uropathy were the commonest causes of AKI (43.75\%). Overall mortality in surgical causes was $43.75 \%$. Among surgical causes maximum mortality was seen in postoperative causes of surgical AKI. But statistically there was no difference in mortality among various surgical causes of AKI. Most of the cases were treated conservatively (68.05\%). The difference in mortality with various modalities of treatment i.e. conservative, peritoneal dialysis and hemodialysis was statistically significant. Mortality was more in patients who needed dialysis. Mortality was more in patients 
treated with peritoneal dialysis than in patients treated conservatively and with hemodialysis. This was because in our institute peritoneal dialysis was considered in patients who were hemodynamically unstable for hemodialysis. Delay in the initiation of treatment was found to be important factor in deciding outcome of AKI. Patients who received treatment after 48 hours of onset of AKI had higher mortality than those who received treatment within 48 hours. Our institute is the tertiary referral centre. Patients are referred from various primary and secondary canters to our institute; therefore there was delay in the treatment of AKI. Peak serum creatinine $\geq 3 \mathrm{mg} \%$ and peak blood urea $\geq 100 \mathrm{mg} \%$ were associated with high mortality rate. In our institute patients come mainly from poor socioeconomic strata, therefore they were not affording for costly investigations like biomarkers of acute kidney injury. Hence peak serum creatinine and peak blood urea levels were still the primary investigations for prognostic indicators of AKI.Hypotension, Bleeding tendency were found to be significantly associated with high mortality. Presences of hyponatremia, hyperkalemia, increased serum bilirubin $(\geq 2 \mathrm{mg} \%$ ), low platelet count $(\leq 50,000)$, leucocytosis were not found to be predictor of high mortality. Other organ involvement in the form of respiratory, liver, CNS and cardiac was associated with high mortality. Most of the cases had complete recovery from AKI. Thus the only factors which were statistically significant in deciding mortality were peak serum creatinine $\geq 3 \mathrm{mg} \%$, peak blood urea $\geq 100 \mathrm{mg} \%$, oliguric AKI, delay in initiation of treatment after onset of AKI ( $\geq 48$ hours), bleeding tendency, hypotension, need of dialysis and associated other organ involvement. Thus most cases of the AKI are reversible, if the etiology is identified and treated early.

\section{Reference}

[1]. Sushrut S Waikar, Joseph V Bonventre : Acute Kidney Injury, Harrison's Principles of Internal Medicine, 18th ed., Fauci et al (eds), McGraw Hill, PP. 2293-2308.

[2]. Kidney International Supplements (2012) KDIGO 2, 8-12.

[3]. Fernando Liano, Julio Pascual and the Acute Renal Failure Study Group. Epidemiology of Acute Renal Failure: A Prospective, Multicenter, Community based Study. Kidney International, Vol. 50 (1996): 811-818.

[4]. Hugh R Brady, Gary G Singer: Acute Renal Failure. The Lancet, vol. 346 December 1995: 1533-1539.

[5]. SL Chew, RL Lins, R Daelemans and ME DeBroe: Outcome in Acute Renal Failure. Nephrology Dialysis Transplantation (1993) 8:101-107. 